Témoigner Témoigner. Entre histoire et mémoire

Getuigen Revue pluridisciplinaire de la Fondation Auschwitz

$116 \mid 2013$

Voyages mémoriels

\title{
Tourisme de mémoire, usages et mésusages : le cas de la Première Guerre mondiale
}

Memory Tourism, use and misuse: the case of the First World War

Herinneringstoerisme, gebruik en misbruik: de Eerste Wereldoorlog

Laurence van Ypersele

(2) OpenEdition

Journals

Édition électronique

URL : http://journals.openedition.org/temoigner/267

DOI : 10.4000/temoigner.267

ISSN : 2506-6390

Éditeur :

Éditions du Centre d'études et de documentation Mémoire d'Auschwitz, Éditions Kimé

Édition imprimée

Date de publication : 1 septembre 2013

Pagination : 13-21

ISBN : 978-2-84174-643-9

ISSN : 2031-4183

Référence électronique

Laurence van Ypersele, «Tourisme de mémoire, usages et mésusages : le cas de la Première Guerre

mondiale », Témoigner. Entre histoire et mémoire [En ligne], 116 | 2013, mis en ligne le 01 juin 2015, consulté le 23 octobre 2020. URL : http://journals.openedition.org/temoigner/267 ; DOI : https:// doi.org/10.4000/temoigner.267 


\section{Tourisme de mémoire, usages et mésusages : le cas de la Première Guerre mondiale}

LAURENCE VAN YPERSELE

Université catholique de Louvain
Acte de la journée d'étude du 21 février 2013 :

"Quand le tourisme questionne la mémoire » organisée par l'asbl Tourisme autrement.

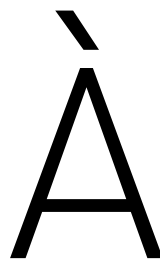

l'aube du centenaire de la Première Guerre mondiale, il me paraît utile d'évoquer la mise en place d'un tourisme de mémoire, les débats que ce tourisme a immédiatement suscités et les ambiguïtés que ce type de tourisme révèle.

Sans doute, ce n'est pas la Première Guerre qui a inventé le tourisme de champs de bataille. Déjà Waterloo, au lendemain de la défaite napoléonienne, avait attiré - et attire toujours - des curieux, des écrivains et des autorités en tout genre. De même les champs de bataille de la Guerre de Sécession aux États-Unis. Mais avec la Guerre 1914-1918, il est clair que l'on change à la fois d'échelle et de contexte culturel. En effet, la puissance de feu provoque dès les premiers jours de guerre des destructions immédiatement perçues comme des atteintes au patrimoine national : l'incendie de la Bibliothèque de Louvain le 25 août 1914 et la destruction de la cathédrale de Reims en septembre 1914 sont à verser au dossier des « atrocités culturelles » commises par l'ennemi. Tandis que les champs de bataille deviennent les lieux du sacrifice national, lieux de terreur et de mort, lieux sacralisés par le sang versé pour la Patrie. Lattachement au patrimoine comme élément d'identité collective et l'attachement à la vie individuelle sont deux éléments autour desquels vont se structurer la mémoire de guerre et le tourisme qui en découle.

\section{LES RUINES ET LES CHAMPS DE BATAILLE, ALLÉGORIES DU CORPS DES COMBATTANTS ET DE LA DOULEUR DE LA NATION}

La puissance de l'artillerie provoque dès août 1914 et jusqu'à la fin de la guerre des destructions inouïes : non seulement les places fortifiées et les ponts stratégiques 
sont atteints, mais aussi les villes et les villages, les maisons, les industries et les églises. Immédiatement, la propagande alliée s'est emparée des photos de ces ruines pour en faire une arme de propagande. À travers les images du désastre, il s'agissait de dénoncer la barbarie teutonne, la violence gratuite de l'ennemi et sa volonté d'atteindre l'identité même de ses adversaires à travers la destruction de son patrimoine. Cette dénonciation permettait de mobiliser les esprits et de légitimer la poursuite de la guerre. La contre-propagande allemande cherchera à répondre en accusant les alliés de certaines destructions, en montrant les édifices et les œuvres d'art non détruits et en rappelant les nécessités de guerre. Tout au long du conflit, des centaines de milliers de photos de ruines et de paysages dévastés seront éditées dans la presse ou sous forme de cartes postales. Les ruines sont également évoquées dans les carnets de soldats. Ainsi, pour ne prendre qu'un exemple, Max Deauville, alias Maurice Duwez, médecin belge et volontaire de guerre, évoque les ruines de Dixmude en novembre 1914 :

Pauvre vieille église de Dixmude, si noble, cachée entre des maisons tranquilles, dans la petite ville déchue, silencieuse, tu dormais, calme, dans tes merveilles. [...] Maintenant, comme les thorax rongés montrent leurs côtes, tu étales tes murs nus, et pour rappeler ta splendeur passée, seules se tordent sur le sol pulvérulent les ferrailles de tes grilles parmi les éclats de tes verrières ${ }^{1}$.

Comme l'a récemment montré Emmanuelle Danchin², ces images sont devenues pour les contemporains des allégories du sacrifice national, métaphore de la mort au combat si difficile à montrer et de la douleur collective si difficile à exprimer : les bâtiments sont des corps éventrés et les paysages, des corps mutilés.

\section{LES PREMIERS VISITEURS}

Autrement dit, dès le début de la guerre, les ruines sont sacralisées et héroïsées : fascination et terreur s'entremêlent. Car les ruines de guerre n'évoquent pas le temps long des ruines classiques, la splendeur d'un temps révolu et des civilisations perdues. Elles évoquent, au contraire, le temps court, celui de la guerre présente et de la violence des hommes ${ }^{3}$. Vivants témoins de l'énormité de l'événement en train de se dérouler. Les zones de combats qui avaient été couvertes par des reporters et des photographes vont, dès la stabilisation du front, attirer toutes sortes de visiteurs: d'abord les militaires allemands qui occupent Liège ou Lille, par exemple, et qui reviennent sur les lieux où ils ont combattu, ensuite des réfugiés qui rentrent inquiets pour constater les dégâts comme à Dinant ou Louvain, ainsi que ceux qui continuent de rendre compte de la guerre - écrivains, peintres, autorités locales et autres - , enfin de simples

(1) Max Deauville, Jusqu'à l'Yser, Louvain, Rex, 1934, p. 224-225.

(2) Emmanuelle Danchin, Les Ruines de guerre et la nation française (1914-1921), Thèse de doctorat, Paris Ouest Nanterre La Défense, 2012.

(3) Emmanuelle Danchin, Les Ruines de guerre et la nation française (1914-1921), op. cit., p. 36-37. 
curieux qui ramassent des souvenirs ou pillent. Par exemple, en novembre 1914, un journaliste de L'Illustration rentrant d'une visite dans la Marne note qu'il a vu des « curieux, pillards malfaisants [...], historiens avides de savoir, malheureux émigrés surtout, ardemment impatients de voir si la chère maison hâtivement abandonnée à l'approche des Allemands est encore debout ${ }^{4}$. » Mais, il n'y a pas que les journalistes et les curieux, les autorités aussi vont visiter ce qu'elles peuvent. En Belgique, par exemple, le cardinal Mercier, de retour de Rome ${ }^{5}$, visitera son diocèse de fond en comble pour se faire une idée du drame vécu par ses fidèles en son absence. Dans sa fameuse lettre pastorale « Patriotisme et endurance » éditée le $1^{\text {er }}$ janvier $1915^{6}$, il commence d'ailleurs par évoquer le choc que fut pour lui la vue de Louvain détruite. Dès la première page, il écrit : « À Rome même j’appris, coup sur coup, la destruction partielle de la collégiale de Louvain, l'incendie de la bibliothèque et d'installations scientifiques de notre grande Université, la dévastation de la ville, les fusillades, les tortures infligées à des femmes, à des enfants, à des hommes sans défense. » Quelques pages plus loin, il revient sur le sujet :

Dans cette chère cité louvaniste, dont je ne parviens pas à détacher mes souvenirs, la superbe collégiale de Saint-Pierre ne recouvrera plus son ancienne splendeur ; l'antique collège Saint Ives ; l'école des beaux-arts de la ville ; l'école commerciale et consulaire de l'Université, les halles séculaires, notre riche bibliothèque avec ses collections, ses incunables, ses manuscrits inédits, ses archives ; la galerie de ses gloires depuis les premiers jours de sa fondation, portraits des recteurs, des chanceliers, des professeurs illustres, au spectacle desquels, maîtres et élèves d'aujourd'hui s'imprégnaient de noblesse traditionnelle et s'animaient au travail : toute cette accumulation de richesses intellectuelles, historiques, artistiques, fruit de cinq siècles de labeur, tout est anéanti ${ }^{7}$

La place particulière du cas de Louvain dans ce texte s'explique notamment par le fait que Désiré Mercier a été professeur à l'Université de Louvain, qu'il y a fondé l'Institut supérieur de Philosophie et qu'il y compte nombre d'amis.

Ces premières visites permettent donc à la fois de prendre conscience de la violence de guerre et de faire le bilan de ce qu'il faudra mettre en ouvre pour la reconstruction. D'autant que, très tôt, l'idée d'une dette envers les cités martyres et les lieux de combat s'impose aux autorités comme aux populations. En France, on

(4) H.G., «Sur les Ruines », in L'lllustration, n 3742, 21 novembre 1914, p. 12 ; cité par Emmanuelle Danchin, Ibid., p. 172.

(5) Le cardinal Mercier était à Rome les premiers jours de guerre pour l'élection du pape Benoît XV. Cf. Jan De Volder, Benoit XV et la Belgique durant la Grande Guerre, Rome/Bruxelles, Institut historique belge de Rome ; coll. « Bibliothèque de l'Institut historique belge de Rome »; n²41, Brepols, 1996.

(6) Les Allemands interdirent immédiatement la lecture de cette lettre dans les églises belges et confisquèrent 40000 exemplaires imprimés. Pourtant, le texte fut traduit dans une demi-douzaine de langues et continua en territoires occupés d'être reproduit sous le manteau jusqu'à la fin de la guerre.

(7) Cardinal Désiré Joseph Mercier, Patriotisme et endurance. Lettre pastorale de S.E. le Cardinal Mercier, Archevêque de Malines, au clergé et aux fidèles de son Diocèse, Belgique, Imprimerie P. Jacquemotte, $1^{\text {er }}$ janvier, Paris, Bloud et Gay, 1915, p. 7. 
légifère dès le 26 décembre 1914 en proclamant « le principe du droit à réparation des dommages matériels subis pour fait de guerre ${ }^{8}$.» Cette loi sera complétée par un décret du 20 juillet 1915 qui consacre le droit à réparation. On songe également au futur redressement économique des régions dévastées. Ainsi, en France, dès la fin 1914, on songe au tourisme - en pleine expansion depuis le dernier tiers du XIXe siècle - pour relancer l'économie, même si le tourisme de champs de bataille n'est pas encore la priorité. Mais, dès 1915, le journaliste Henri Lavedan souhaite que des visites soient organisées pour rendre hommage aux sacrifices consentis et célébrer la nation souffrante :

Nous choisirons [...] les régions martyrisées ou simplement meurtries. Si nous ne pouvons
pas encore y accéder, nous nous avancerons jusqu'au point extrême où l'on aura licence
d'approcher, afin de nous sentir le plus près des blessures que nous aurons la douleur
d'être impuissants à guérir. [...] Il ne s'agira pas là d'une promenade pareille à celles
d'autrefois. Nous serons en plein air dans un temple immense et comme illimité, où finità
peine de célébrer, ainsi qu'une magnifique et sanglante messe, le sacrifice de la guerre'.»

Le tourisme de guerre relèvera donc du pèlerinage patriotique, du sentiment religieux laïcisé, de la communion nationale. Et, en effet, en septembre 1915, des membres du Parlement, les autorités locales et de simples civils vinrent commémorer le premier anniversaire de la bataille de la Marne qui arrêta l'avancée allemande en territoire français. Pour l'occasion, deux monuments furent inaugurés : l'un à Barcy, l'autre à Etrepilly ${ }^{10}$. Avec le repli de l'armée allemande sur la ligne Siegfried, en 1917, les visites officielles, avec remise de drapeaux et décoration des ruines comme si elles étaient des soldats, se multiplient : le Président de la République, des ministres, des sénateurs, des généraux et même des conservateurs de musée viennent ponctuellement honorer ces lieux devenus sacrés.

On trouve la même sensibilité, dès 1915, côté anglais où l'on imagine créer aprèsguerre une voie sacrée traversant toute la ligne du front : «j’aimerais, écrit un officier britannique, obliger tous les hommes, les femmes et les enfants d'Europe de l'Ouest à faire un pèlerinage le long de cette Via Sacra, de façon à ce qu'ils puissent réfléchir et découvrir le sens de la guerre ${ }^{11}$. » Tandis que Winston Churchill, qui a combattu dans les Flandres à Ploegsteert, souhaite que les Anglais rachètent Ypres pour la conserver en l'état et en faire un lieu de pèlerinage et d'hommage aux sacrifices consentis par les Britanniques. En Belgique, la majeure partie du pays étant occupée, la question se pose un peu différemment. Les difficultés de circulation et l'interdiction de toute manifestation patriotique rendent les visites des lieux de combats quasiment

(8) Emmanuelle Danchin, Les Ruines de guerre et la nation française (1914-1921), op. cit., p. 158.

(9) Henri Lavedan, «Les grandes Heures. Les prochains Voyages », in L'lllustration, n 3761, 3 avril 1915, p. 2-3, cité par Emmanuelle Danchin, Les Ruines de guerre et la nation française (1914-1921), op. cit., p. 177.

(10) Emmanuelle Danchin, Les Ruines de guerre et la nation française (1914-1921), op. cit., p. 177.

(11) Cité par Emmanuelle Danchin, Ibid., p. 178. 
impossibles. Toutefois, à la Toussaint, les populations viennent spontanément rendre hommage aux soldats tombés lors de la guerre de mouvement. Ainsi, par exemple, en 1916, Le Vingtième siècle, journal catholique en exil à Paris, note :

Au cimetière d'Uccle, on observe que la tombe d'un soldat inconnu n'est pas moins fleurie que celle de ses camarades. Personne dans la foule n'oublie le tertre de ce héros obscur: L'édilité de Laeken, obéissant à une délicate inspiration, a fait dresser une table de marbre imprimé sur laquelle sont inscrits les noms de tous les soldats de Laeken tombésà l'ennemi et dont beaucoup reposent dans les plaines désolées de l'Yser. C'est par brassées que les visiteurs ont jeté des fleurs au pied de ce mémorial ${ }^{12}$.

On le voit l'idée de pèlerinage est précoce et implique d'emblée une réflexion sur la préservation des lieux.

\section{DÉBATS SUR L'AVENIR DES RUINES ET DES CHAMPS DE BATAILLE}

Au départ, l'idée de conserver certaines ruines comme témoignage à la fois du vandalisme teuton et de l'héroïque sacrifice allié s'impose à l'unanimité. Ainsi, lit-on en 1915 dans le Courrier de l'Armée à propos de Visé :

À notre avis, il n'y a pas à hésiter, il ne faut pas toucher à Visé ; [...] il faut que l'on veille sur ses ruines ; il faut que Visé apprenne au monde la barbarie teutonne; il faut que Visé soit une leçon de tous les jours, où les Belges viendront chercher les aliments pour entretenir la haine sacrée et où les étrangers contempleront les bienfaits de la Kultur germanique. Quant à Visé, la cité vivante, on la reconstruira, en aval, entre les ruines actuelles et ce qui reste du village de Mouland ${ }^{13}$. De même en France, on propose de conserver « pieusement » certains villages détruits, des monuments historiques et des champs de bataille pour, dit Georges Reynald au Sénat en 1915, que « nous ne puissions oublier, pour que nos enfants se souviennent, pour que les autres nations comprennent et jugent, il convient que la France garde au visage la cicatrice de ses blessures ${ }^{14}$. » Toutefois, la conservation en l'état des ruines et des paysages est très onéreuse et pose des problèmes quasiment insurmontables : pour les ruines, le temps continuera son œuvre de désagrégation ; tandis que pour les paysages, la nature reprendra ses droits ; sans compter le désir des habitants de retrouver une vie normale après-guerre. Aussi bien, réévaluera-t-on, durant toute la guerre, la liste des ruines à conserver, jusqu’à la réduire à peau de chagrin au lendemain du conflit.

(12) « La population de la Capitale a honoré les sépultures de nos soldats », in Le Vingtième Siècle, Paris, 14 novembre 1916, p. 2.

(13) P. Sambrée, «La Reconstruction de nos cités », in Courrier de l'Armée, no 152, 26 août 1915, p. 2. Il y eu des propositions semblables pour Ypres, Arras et Reims.

(14) Cité par Philippe Chassaignen, Jean.-Marc Largeaud (dir.), Villes en guerre, Paris, Armand Colin, 2004, p. 13. 
La reconstruction des habitations et des villes suscitera, là aussi dès le début de la guerre, des débats sans fin. En effet, différentes positions s'affrontent. Les uns sont partisans d'une reconstruction à l'identique, particulièrement pour les ensembles architecturaux anciens comme la Grand Place d'Arras ou la place du Vieux Marché à Louvain. D'autres, notamment des architectes, sont partisans d'une modernisation : il s'agit de profiter de l'occasion pour faire du neuf. D’autres encore, à mi-chemin entre ces deux positions, prônent une reconstruction à l'ancienne qui introduirait tout de même une modernisation (en termes d'électricité et de chauffage, par exemple) ${ }^{15}$.

\section{LES PREMIERS GUIDES TOURISTIQUES}

Les opérateurs touristiques s'engagent, eux aussi, dans ces débats. Ainsi, le Touring Club de France se mobilise durant toute la guerre pour appeler au relèvement des régions dévastées par le tourisme. Car le tourisme impliquerait un réaménagement des routes, susciterait des emplois et accélérerait le relèvement de l'habitat. De même, André Michelin - qui édite des guides touristiques depuis 1900 - pense, dès 1916, que les zones de combat qui suscitent déjà des pèlerinages deviendront des lieux touristiques importants après-guerre. Ainsi, décide-t-il de lancer une série de guides consacrés aux champs de bataille. Son but est double : participer au relèvement national par le tourisme et évincer ses concurrents. Le premier guide paraît en septembre 1917 pour le troisième anniversaire de la bataille de la Marne ${ }^{16}$. De nombreux autres suivront.

\section{LA SORTIE DE GUERRE}

Si les premiers parcours touristiques sont pensés pendant la guerre, il faut attendre la fin du conflit pour qu'ils soient réellement mis en œuvre. Au lendemain de l'armistice, ces espaces vantés par les guides touristiques et les affiches sont visités par les anciens combattants, les familles endeuillées, les enfants des écoles et de simples curieux. Le front est à la fois un espace sacré et un espace profane. «Pour les anciens combattants, retourner sur le champ de bataille, c'est revenir, c'est se souvenir, c'est se confronter à son passé, voire exorciser la guerre et c'est parfois aussi rendre hommage à un camarade tué17. » Pour les familles endeuillées, c'est se recueillir sur le lieu de la mort d'un proche, c'est le garder vivant dans les mémoires. En revanche, pour les curieux, c'est confronter les images qu'ils se sont faits de la guerre et la réalité des champs de bataille. Dès le départ, une distinction apparaît donc entre pèlerin et touriste. Le pèlerin vient légitimement se recueillir sur une terre sacrée. Le touriste, assimilé aux embusqués de l'arrière, vient assouvir une curiosité malsaine et profaner les lieux par leur comportement irrespectueux, comme en témoigne cet article paru en juin 1919:

(15) Cf. Emmanuelle Danchin, Les Ruines de guerre et la nation française (1914-1921), op. cit., p. 160-165.

(16) Ibid., p. 259.

(17) Ibid, p. 320. 
Une bonne partie des « pèlerins » est recrutée dans la foule des désœuvrés ou des snobs qui vont là comme ils iraient aux courses. Leur nature gouailleuse et turbulente prend le dessus et leur attitude devient une profanation pour nous. Comment voulez-vous leur demander de rester une journée recueillis et constamment pénétrés de la tragique grandeur d'un monotone néant où rien ne parle ? [...] Ils s'attachent à un panorama de la Grande Guerre, frappante image, mais que peuvent leur dire des pierres accumulées sur des pierres, des trous voisinant avec d'autres trous sur le sol parsemé de débris misérables de caisses et épaves sans nom ${ }^{18}$ ?

Mais le front, c'est aussi un espace de vie pour les habitants qui y sont restés ou qui reviennent. Ni pèlerins ni touristes, ces civils sont avant tout préoccupés par un quotidien à reconstruire, une maison à réparer ou à rebâtir, une ferme à remettre en état, un commerce à relancer. D’ailleurs, les habitants des régions sinistrées sont partagés sur les bénéfices et les inconvénients que constitue cet afflux de touristes. D’un côté, les sinistrés dont la vie quotidienne est particulièrement misérable ne se sentent pas soutenus et respectés « par ceux qui ne sachant que faire de leur dimanche, s'en allaient faire une partie de campagne dans nos ruines et rentraient le soir satisfaits d'avoir passé une bonne journée ${ }^{19}$. » De l'autre, ils voient le tourisme à la fois comme un moyen de faire connaître leurs souffrances qui ne doivent pas être oubliées et comme un élément de relance économique qui favorisera la reconstruction. Encore faut-il organiser ce tourisme dans le sens des intérêts locaux, en réglementant l'afflux de voyageurs et en créant des centres hôteliers adaptés ${ }^{20}$. Le Touring Club de France, comme le Touring Club de Belgique s'y emploieront. Les guides touristiques comme Michelin et les agences de voyage comme Thomas Cook \& Son proposèrent, eux aussi, des circuits touristiques très précis qui tenaient compte de l'accessibilité des lieux et de leur dangerosité. Les ruines, les monuments et les cimetières étaient autant de points de repère pour se diriger au milieu des paysages dévastés. En 1919 et 1920, ce sont des centaines de milliers de visiteurs français, belges, britanniques et américains qui parcoururent le front depuis le Westhoeck jusquà l'Alsace. La popularité de ces visites témoigne du besoin de voir la guerre, de se souvenir des atrocités commises par l'ennemi et de rendre hommage aux morts.

\section{ET APRÈS...}

La victoire changea le rapport que l'on entretenait avec les destructions. Si certains souhaitaient encore garder des ruines et des paysages en l'état, les habitants des régions meurtries souhaitent un retour à la vie et la population en général considérait de plus en plus la reconstruction comme le parachèvement de la victoire. Dès lors, les

(18) Le Journal des régions dévastées pour la défense des Sinistrés du Nord et de l'Est, no 7, 24 juin 1919, p. 108-109.

(19) Louis Revault (député de la Meuse), «le Tourisme dans les pays dévastés? », in Le Journal des régions dévastées pour la défense des Sinistrés du Nord et de l'Est, n5, 15 juin 1919, p. 68.

(20) Cf. Emmanuelle Danchin, Les Ruines de guerre et la nation française (1914-1921), op. cit., p. 327. 
Halles de Louvain et celles d'Ypres, la Grand Place d'Arras et la cathédrale de Reims furent reconstruites à l'identique. Certains quartiers, comme certaines églises furent rebâtis dans des styles modernes. Un peu partout, les maisons furent modernisées. Seuls quelques villages complètement rasés, notamment près de Verdun, restèrent en ruines. De même, les paysages dévastés ne pouvaient rester en l'état : déminés, ils furent remis en culture ; conservés, comme à Verdun, ils reverdirent. Dès le début des années 1920, les anciens combattants qui revenaient sur les champs de bataille ne reconnaissaient plus les lieux où ils avaient tant souffert. On conserva néanmoins quelques vestiges de guerre : le fort de Loncin à Liège, le boyau de la mort à Dixmude, les tranchées de Beaumont-Hamel dans la Somme, celles de l'Hartmannswillerkopf en Alsace, etc.

Par ailleurs, à partir de 1920, tous les belligérants, sauf l'Empire britannique, décidèrent de rapatrier les corps des soldats. En Belgique, par exemple, c'est à la demande des familles et aux frais des communes que ces rapatriements ont lieu. Sur le front, de grands cimetières et des ossuaires sont réorganisés pour les soldats britanniques, ainsi que pour tous les soldats non identifiés ou non réclamés. Au même moment, on assiste à une vague d'érection de monuments aux morts un peu partout en Europe, voire au-delà. Ces monuments ramènent le souvenir de chaque mort dans les villes et les villages qui, souvent, n'ont connu la guerre que de loin. Tandis que l'inhumation d'un Soldat inconnu dans toutes les capitales belligérantes, à l'exception de Berlin et Moscou, doit permettre à tous les endeuillés de trouver un lieu où se recueillir. Or, que ce soient les cimetières militaires sur les champs de bataille avec leurs alignements parfaits ou les monuments aux morts dans les villages avec leur statue de soldat mourant dans un uniforme impeccable, sans boue ni blessure, partout la violence de guerre et la mort au combat sont aseptisées. En termes de tourisme, ce sont désormais les cimetières réaménagés sur les champs de bataille et les grands mémoriaux - notamment aux disparus - que les parcours mettent en évidence; des lieux qui permettent un recueillement plus apaisé parce qu'ils passent par un certain oubli de la brutalité de guerre.

En tous cas, le rapatriement des corps et de la mémoire de guerre dans les lieux d'origine rendit les pèlerinages moins nécessaires pour les familles endeuillées. Désormais, le souvenir des «Grands Morts » se vit et se transmet autour du monument aux morts local ou au pied du Soldat inconnu. Un souvenir toutefois qui s'est peu à peu effacé devant celui de la Seconde Guerre mondiale. Seuls les Britanniques, les Australiens, les Néo-Zélandais et les Canadiens continuent, jusqu’à aujourd'hui, à se rendre en pèlerinage sur la tombe de leurs ancêtres ou devant les grands mémoriaux (la porte de Menin à Ypres, le mémorial de Thiepval ou celui de Villers-Bretonneux dans la Somme) pour rendre hommage à ceux qui se sont sacrifiés pour leur nation. Les pèlerinages de l'Yser, quant à eux, rassemblent depuis 1921 les nationalistes flamands autour du souvenir des soldats flamands sacrifiés en vain. Ainsi, la tour de l'Yser, gigantesque monument à la paix, inauguré en 1930, est en même temps le symbole du combat flamingant. Cela étant, pour les Belges et les Français, la visite des lieux de combat relève plutôt d'un tourisme de champ de bataille qui s'enracine aussi bien 
dans la fascination pour la violence que dans le désir de découvrir ou redécouvrir un passé désormais lointain.

\section{CONCLUSION}

L'approche du centenaire de la Première Guerre mondiale relance la réflexion autour du tourisme de mémoire, comme on l'appelle aujourd'hui. Que ce soit en France, en Flandre ou en Wallonie, tous les plans concernant les commémorations 1914-1918 ont un important volet touristique. En Flandre, c'est même le ministre du Tourisme qui pilote les préparatifs. Comme par le passé, ce tourisme s'articule autour d'un triple objectif : la préservation et la transmission d'un héritage historique, la valorisation du patrimoine civil et militaire qui doit alimenter la notoriété régionale et le développement économique des régions concernées. Mais, un siècle plus tard, au moment où il n'y a plus aucun témoin, le contenu de ces objectifs a fortement évolué. Sur le plan économique, on comptait sur le tourisme pour reconstruire les régions dévastées, alors qu'aujourd'hui il s'agit de développer le commerce et d'aménager les territoires : de nouveaux musées sortent de terre comme à Ploegsteert ou à Mons, d'autres sont rénovés comme à Ypres et à Péronne, l'hôtellerie d'Ypres d'ailleurs se prépare à accueillir des centaines de milliers de visiteurs en 2014. Sur le plan de la notoriété, l'urgence était de ne pas être oublié, alors qu'aujourd'hui on cherche une visibilité internationale. Les projets de classement de certains champs de bataille par l'UNESCO sont symptomatiques à cet égard. Sur le plan de la transmission mémorielle, enfin, on souhaitait se souvenir du sacrifice national pour la victoire de la civilisation contre la barbarie, alors qu'aujourd'hui il s'agit surtout de transmettre un message de paix, voire de réconciliation, de dénoncer l'horreur de la guerre, de commémorer les victimes plutôt que les héros, mais aussi de retrouver un passé oublié. Les touristes viendront chacun avec leurs attentes particulières : sentimentalisme, curiosité intellectuelle, désir de ressentir la guerre, quête des ancêtres, affirmation identitaire, obligation scolaire... Les uns, comme nombre d'Australiens, viendront en pèlerinage rendre hommage à ceux qui ont fait naître leur pays à lui-même. D'autres, comme nombre d'écoliers, viendront apprendre un passé lointain qui est censé les concerner, passé glorieux comme à Loncin, passé terrifiant comme à Ethe ou à Verdun, passé toujours triste comme dans les innombrables cimetières qui parsèment le front. D'autres encore se plongeront avec fascination dans la puissance destructrice de la guerre et dans l'horreur des tranchées, encore qu'il faille de l'imagination pour ressentir l'horreur de la guerre devant les vestiges des tranchées. En fait, les touristes projetteront leur propre imaginaire de guerre, façonné par leur éducation, par les musées et par les visites guidées (d'où l'importance de ceux-ci), sur les ruines, les monuments, les cimetières qui à eux seuls ne parlent guère. 